\title{
Téoros
}

Revue de recherche en tourisme

\section{Paradigme d'écotourisme et sociétés traditionnelles en mutation}

\author{
Le cas de l'outre-mer français
}

\section{Jean-Marie Breton}

Volume 23, numéro 2, été 2004

URI : https://id.erudit.org/iderudit/1071310ar

DOI : https://doi.org/10.7202/1071310ar

Aller au sommaire du numéro

Éditeur(s)

Université du Québec à Montréal

ISSN

0712-8657 (imprimé)

1923-2705 (numérique)

Découvrir la revue

Citer cet article

Breton, J.-M. (2004). Paradigme d'écotourisme et sociétés traditionnelles en mutation : le cas de l'outre-mer français. Téoros, 23(2), 54-60.

https://doi.org/10.7202/1071310ar d'utilisation que vous pouvez consulter en ligne.

https://apropos.erudit.org/fr/usagers/politique-dutilisation/ 


\title{
Paradigme d'écotourisme et sociétés traditionnelles en mutation
}

\author{
Le cas de l'outre-mer français
}

\section{Jean-Marie Breton}

Le tourisme est fréquemment présenté comme une alternative d'impulsion et de financement d'un développement que, depuis peu, l'on veut par définition «durable», au point d'en générer une expansion aussi difficilement maîtrisable qu'incohérente, au détriment du patrimoine environnemental, insulaire et littoral en particulier, comme c'est le cas dans les DOM-TOM français'. Bien commun de tous et ancrage des cultures comme des identités, ce patrimoine en subit des atteintes et des agressions particulièrement graves, en termes de dégradations multiples, voire de destructions irréversibles.

La soumission dès lors nécessaire et incontournable de l'activité touristique aux exigences de protection, de conservation et de gestion valorisante et reproductible de la biodiversité et du patrimoine naturel, à travers sa compatibilité et sa mise en cohérence avec les réglementations protectrices de l'environnement, est de nature à générer des approches, des stratégies et des comportements nouveaux. C'est le cas en matière de politiques publiques comme de gestion opérationnelle, favorables tant à un développement durable des sociétés qu'à l'optimisation du cadre et de la qualité de vie des hommes. Le «développement touristique durable» apparaît alors comme un sous-ensemble d'un développement global à dimension holistique.

La démarche écotouristique répond à ces préoccupations et constitue un défi stimulant pour les opérateurs du tourisme comme pour les acteurs de l'environnement, sinon une opportunité de «réappropriation» de leur milieu de vie par les populations locales. Il convient alors d'en clarifier le concept, eu égard à la signification et à la portée controversées de sa dimension «paradigmique »; avant d'en déterminer la validité dans l'outre- mer français, au regard des défis et des enjeux d'une gestion participative des ressources touristiques au service d'un développement durable et viable.

\section{L'écotourisme comme paradigme : un concept controversé ${ }^{2}$}

Le concept

\section{Les différentes approches}

Nombreuses - trop nombreuses ! - sont aujourd'hui les définitions de l'écotourisme, qui se superposent, s'enchevêtrent, parfois même se contredisent et s'excluent. Il serait aussi inutile que vain d'en refaire ici l'historique ou de tenter d'en explorer l'énumération. On se bornera à cet égard à quelques repères et références basiques, à défaut de pouvoir se prétendre essentiels.

P. Py (2000 : 41) s'est efforcé d'expliciter la relation du tourisme écologique à l'écotourisme et d'en éclairer les contours. Alors que le premier peut être défini comme «un tourisme non agressif à l'égard de l'environnement tant naturel et urbain que social», il voit dans le second, qui en serait alors un avatar particulier, une forme de tourisme responsable, non pas en l'occurrence un produit, mais au contraire «un mode de développement du tourisme » qui se déterminerait par rapport à quatre objectifs déterminants : respect mutuel entre touristes et hôtes, participation active des populations, valorisation des sociétés d'accueil et découverte de celles-ci par les touristes.

Utilisé à l'origine pour désigner un tourisme de nature, le concept a ensuite permis d'appréhender un tourisme naturaliste d'observation et d'étude de la faune et de la flore dans les zones protégées, vierges et non perturbées par l'homme et offrant une grande diversité biologique (Blangy, 1993: 223).
Certains y ont alors vu «un secteur du tourisme impliquant la visite de sites naturels relativement intacts avec le seul et unique objet d'admirer, d'étudier et de profiter de la beauté des lieux, de ses plantes et animaux sauvages, et de toutes les spécificités culturelles qu'on peut y découvrir» (Kurt, 1993 : 214); alors que, pour d'autres,

l'écotourisme ne peut pas être considéré comme un simple secteur d'activité de l'industrie touristique [dès lors que] les fondements éthique et philosophique de l'écotourisme englobent toutes les facettes d'un tourisme responsable, tant du point de vue social qu'écologique [...] Il s'agit en fait d'un modèle évolutif de développement durable dans lequel les besoins économiques, sociaux et esthétiques sont satisfaits tout en préservant l'intégrité culturelle, les processus écologiques essentiels, la biodiversité et les cycles vitaux (ibid.).

Des tentatives de systématisation de la notion, à partir des définitions successives du concept, n'ont qu'en partie contribué à l'éclairer et à en cerner, sinon a minima, les contours et la portée (Blangy, et al., 2002). On a logiquement cherché des réponses dans les relations du tourisme à l'environnement, dans une approche dynamique qui entend aller au-delà de définitions par essence trop réductrices et donc, partant, imparfaites. L'écotourisme se situerait ainsi au double carrefour des politiques publiques du tourisme et de l'environnement, d'une part, du tourisme et du droit de l'environnement, d'autre part (Blouin, 2000a: 25-32).

Si l'on se tourne vers les « institutionnels», les définitions officielles ne sont non plus absentes, dans le souci légitime - mais y sont-ils véritablement parvenus ? - de mieux cadrer le débat, pour pouvoir en induire 
une démarche pragmatique et opérationnelle. Selon l'International Ecotourism Society, l'écotourisme ${ }^{3}$ constitue " une forme de voyage responsable, dans les espaces naturels, qui contribue à la protection de l'environnement et au bien-être des populations locales » (Blangy, 1993 : 13). L'OMT, pour sa part, a entendu distinguer formellement le tourisme de nature de l'écotourisme, voyant dans ce dernier une forme de tourisme qui regroupe des caractéristiques expressément énumérées.

Lors du Sommet mondial de l'écotourisme, tenu à Québec en mai 2002 à l'occasion de l'année mondiale qui lui était consacrée, ce même organisme a contribué à la formalisation de constats et de recommandations contenus dans la Déclaration de Québec sur l'Écotourisme ${ }^{4}$. Celle-ci a permis d'affiner l'approche conceptuelle et dynamique de la notion, approche qui, selon certains, constituerait désormais la référence de base dûment clarifiée. Sans doute serait-il plus pertinent et réaliste, compte tenu des réserves que cette manifestation et, partant, ce texte, peuvent susciter $^{5}$, d'y voir un point de départ plutôt qu'une définition achevée du concept ${ }^{6}$.

D'aucuns n'ont pas manqué depuis lors de continuer à y voir, à juste titre, «un phénomène relativement complexe, en évolution, qui ne s'appuie sur aucune définition universellement reconnue (Couture, 2002a: 3). Ils soulignent à cet égard que les définitions les plus récentes ou les plus largement admises sont celles qui préconisent une approche davantage normative et s'insèrent dans une philosophie de développement durable et responsable du tourisme en milieu naturel (Couture, 2002b : 6).

\section{La construction du paradigme ${ }^{7}$}

Des auteurs, québécois en particulier, se sont attachés à étudier et à mettre en lumière les différents aspects et problématiques de la «gouvernance participative » des ressources de biodiversité affectées au tourisme (Lequin, 2001), ou de la participation des communautés locales au développement durable et au tourisme viable ${ }^{8}$. Ils voient dans l'écotourisme un «nouveau paradigme», dont ils s'attachent alors à construire le contenu et la signification à partir d'une analyse du concept, de ses définitions et de ses impacts.

La justification de la démarche réside en l'espèce dans la considération qu'il s'agit du secteur de l'industrie de voyage qui connaît la croissance la plus rapide tout en reposant sur une nouvelle approche qui allie les objectifs de protection (des zones naturelles menacées) à la participation des communautés locales (à leur propre développement). On peut en ce sens considérer que l'écotourisme, malgré les réserves qu'il peut susciter (comme nous le verrons ci-dessous), ajoute au développement du-rable les dimensions du patrimoine culturel et l'inclusion des communautés locales, afin de contribuer à leur bien-être et à un tourisme à l'échelle humaine, principes qui rejoignent l'objectif du tourisme dit social et humaniste (Gagnon, 1999). L'écotourisme doit alors prendre en compte ces deux dimensions de durabilité de la ressource et de participation des populations à un projet de développement (Lequin, $2001: 3)^{9}$.

Ainsi, l'écotourisme peut être appréhendé et compris comme « un effort de collaboration entre les communautés locales, les intervenants touristiques et les protecteurs de la ressource, dont l'État, pour préserver les espaces naturels qui servent de support au développement régional»(ibid.). Un tel type de développement reposerait en ce sens sur trois postulats de base: caractère durable du développement ; absence de définition a priori du développement durable dans un contexte local ou régional exprimant une situation empirique donnée; exigence d'une négociation appropriée entre partenaires, afin de déterminer un équilibre durable entre intérêts écologiques et économiques ${ }^{10}$.

La construction du paradigme de l'écotourisme repose dès lors conjointement sur la recherche de l'origine du concept et sur le recensement de ses définitions. Celles-ci, sur lesquelles nous ne reviendrons pas, procèdent de l'approche et de l'orientation principale retenues, respectivement axées sur la demande, sur la ressource, ou sur la communauté d'accueil ${ }^{11}$. Partant, il importe d'en identifier les effets, à la fois sur les écosystèmes, sur l'économie locale et sur les communautés d'accueil.

\section{La signification du paradigme}

L'équilibre que postule le paradigme d'écotourisme devrait alors être compris, dans le sens évoqué ci-dessus, comme «une tension dynamique et évolutive dans le temps, et non comme une condition fixe de développement [dès lors que] l'écotourisme durable repose sur le processus d'interaction avec l'environnement et les échanges culturels avec les communautés d'accueil» (Lequin, 2001 : 25).
Ce n'est pas le lieu de développer ici une critique du paradigme d'écotourisme, sauf pour dénoncer la connotation inévitablement mythique qui est de nature à affecter la portée réelle du concept et donc sa signification opératoire $^{12}$. Si l'on considère qu'il existe toutefois une relation privilégiée entre écotourisme et développement durable, le premier reste par ailleurs assez largement tributaire de la problématique et de la définition comme des caractéristiques du second, là encore nécessairement polymorphe et relativisé, selon qu'en est retenue une approche écologique, co-évolutive ou politique.

En termes de politiques publiques, un projet d'écotourisme ne peut satisfaire au exigences de développement durable que pour autant qu'il réponde conjointement à des objectifs de durabilité de la ressource, d'équité intergénérationnelle et de sensibilisation des individus à l'environnement. La conciliation attendue ne peut être alors que le résultat d'une négociation entre les différents intervenants au profit de l'équilibre à instaurer entre les intérêts en présence, à partir de l'identification, particulièrement complexe et controversée, des valeurs qui orientent les choix et les outils afférents à la réalisation d'un développement durable ${ }^{13}$.

\section{Le débat}

Les ambiguiltés polysémiques

Il est manifeste que l'accord est loin d'être réalisé sur une définition de la notion d'écotourisme qui puisse rallier les suffrages du plus grand nombre ${ }^{14}$. Là où certains n'y voient qu'un avatar d'une forme de tourisme respectueuse des exigences de protection de l'environnement et de la nature, d'autres en font à l'inverse une démarche spécifique, dont le contenu et la portée diffèrent assez largement de la vision du tourisme classique ${ }^{15}$.

Tourisme durable, viable, solidaire, communautaire, participatif (écologiquement) responsable, équitable, affinitaire, récréotourisme, tourisme «vert», d'aventure, de plein air, culturel et scientifique, etc., les épithètes et les qualificatifs ne manquent pas $^{16}$ pour appréhender l'écotourisme. L'imagination est probablement loin de les avoir épuisés, même si, ici, le déterminant apparaît plus porteur de confusion, à l'endroit du déterminé, qu'il ne contribue à le cerner et à l'éclairer. 
Tourisme de découverte à vocation scientifique, pour les uns, axé sur les valeurs de la nature ${ }^{17}$ et de la biodiversité ; tourisme de rencontre socioculturelle, entre les détenteurs d'un patrimoine et de valeurs qu'ils entendent préserver et valoriser et des hôtes appelés à les découvrir et à les partager, pour les autres.

Or, l'écotourisme ne saurait se réduire pour autant à une simple forme de tourisme à vocation écologique qui, tout en conservant sa dimension et ses perspectives premières, se bornerait à intégrer le respect de l'environnement naturel en s'inscrivant dans le sens d'une plus grande préoccupation de protection, de sauvegarde et de valorisation du patrimoine environnemental ${ }^{18}$.

L'écotourisme procède certes de la démarche écologique, mais dépourvue de sa connotation militante. Il dépasse cette seule dimension au profit d'une perception et d'une vision spécifiques de l'environnement (au sens large retenu) ${ }^{19}$, dans une approche résolument originale. Il serait moins à cet égard un tourisme soucieux ou teinté d'écologie qu'un tourisme qui intègre une démarche culturelle et scientifique à caractère patrimonial ${ }^{20}$.

\section{Le pluralisme conceptuel}

Il en est allé en l'occurrence de l'écotourisme comme de nombreuses notions, qui perdent en rigueur et en signification, au plan sémiologique aussi bien que sémantique, ce qu'elles gagnent en extension, en diversification et, surtout, en vulgarisation dans l'usage qui en est fait. Paré d'un halo qui interdit de la comprendre et d'en cerner la portée, l'écotourisme est aujourd'hui arboré à tout propos et quasi présenté comme la panacée universelle des maux dont souffrirait le tourisme de masse. L'invocation répétée du concept et la médiatisation complaisante du terme (prétexte et/ou alibi d' «autolégitimation»?) se substituent trop souvent au débat et à la réflexion, lorsqu'ils ne les évacuent pas purement et simplement.

La notion est alors inévitablement devenue, refuserait-on de l'admettre, un véritable symbole de confusion, à l'endroit de laquelle les critères et les référents retenus dans la Déclaration de Québec susvisée, au terme du Sommet mondial sur l'écotourisme, n'ont apporté, de notre point de vue, que des réponses partielles et parfois contestables ${ }^{21}$. Cette ambiguïté n'a pas échappé à certains observateurs avertis, qui ne laissent pas entendre autre chose, serait-ce implicitement.
On a ainsi pu souligner

[qu'] aux définitions proposées par des chercheurs, des universitaires et des organisations s'ajoutent celles élaborées par les agences de tourisme de pays, de provinces ou de régions [...]. Les destinations intéressées par cette forme de tourisme [...] ont pour la plupart élaboré leur propre définition ou adopté une définition existante en fonction de leurs besoins ou de leur compréhension du terme (Couture, 2002b: 6-7).

À la fois véritable auberge espagnole, où chacun y met ce qu'il y apporte ${ }^{22}$ - ou ce qui l'arrange ! -, il constitue, comme les fameuses langues d'Ésope, la meilleure des choses et l'alternative déterminante de tous les problèmes, pour les uns, la pire des notions, en raison de l'imprécision, de l'ambiguïté et de l'élasticité à géométrie variable de son contenu, pour les autres (Bouin, 2000b : 417). Sans doute - in medio stat virtus - la réalité se situe-t-elle à mi-chemin de ces deux visions...

\section{La dimension mythique}

Mythe ou paradigme? Slogan ou concept opérationnel? Mode, ou tendance à long terme (Blangy et al., 2002) ? La question mérite d'être posée et, au stade actuel, ne peut plus être esquivée, serait-elle dérangeante. L'arbre du mot ne cacherait-il pas la forêt de la problématique, des implications, des enjeux et, partant, des intérêts, multiples et souvent antagonistes, sous-jacents?

Mythe et paradigme ? On ne peut exclure $a$ priori que la perception intuitive de l'écotourisme ne soit pas apte à générer un paradigme nécessaire, sinon même à constituer une conditionnalité de sa formulation. Le passage du discours à la démarche opérationnelle, essentielle dans son approche économique, n'est-il pas alors le cheminement logique indispensable pour crédibiliser l'offre correspondante et y sensibiliser les consommateurs comme les acteurs potentiels ? Le débat, en tout état de cause, ne saurait être esquivé.

Pour ne pas encourir le reproche de le faire, on peut alors être tenté d'appréhender basiquement l'écotourisme, dans l'approche qui en a été esquissée plus haut, et sous bénéfice d'inventaire, comme un tourisme scientifique et culturel de découverte, d'exploration et de loisirs, respectueux des exigences et/ou compatible avec les contraintes écologiques telles que définies et sanctionnées par les nor- mes juridiques de protection et de conservation de l'environnement dans son acception patrimoniale extensive.

Il convient, en tout état de cause, de se démarquer des ambiguïtés du mythe pour retrouver le pragmatisme et l'efficacité attendus des alternatives opérationnelles que le l'écotourisme peut apporter à la dimension et aux limites d'un tourisme jusqu'alors principalement cantonné à une dimension essentiellement commerciale, c'est-à-dire exposée aux excès d'une logique à dominante économique et productive. La question est alors, entre autres, celle de savoir dans quelles conditions, selon quelles modalités et avec quelles limites une telle démarche serait apte à répondre aux attentes de pays détenteurs d'un large potentiel touristique, source d'une offre importante, mais encore insuffisamment exploitée.

Au-delà d'une recherche, au demeurant légitime, de rentabilité immédiate, il importe, lorsque l'on est en présence de sociétés en mutation encore largement marquées par des valeurs, des dynamiques et des modes de vie traditionnels, de rechercher si les effets pervers induits, ou implicites, dont l'écotourisme n'est pas exempt, ne sont pas de nature à en remettre en cause les avantages par ailleurs escomptés.

La référence à la situation des territoires de l'outre-mer français (comme, dans une certaine mesure, mutatis mutandis, à celle de bon nombre de pays du tiers-monde, le contexte politique serait-il différent) incite à cet égard à une certaine prudence, sinon parfois à une nécessaire circonspection, envers des initiatives, des politiques et des projets que l'on peut être enclin à concevoir et à mettre en œuvre in situ.

\section{L'écotourisme dans l'outre-mer français: les insuffisances fonctionnelles du paradigme}

\section{Les limites de la démarche écotouristique}

\section{Le risque de déstructuration sociale}

Il convient d'être particulièrement prudent quand on envisage la conception et l'exploitation de «produits » écotouristiques au profit de communautés locales marquées par un fort particularisme ${ }^{23}$ en matière de structuration et de cohésion sociales (assises en 


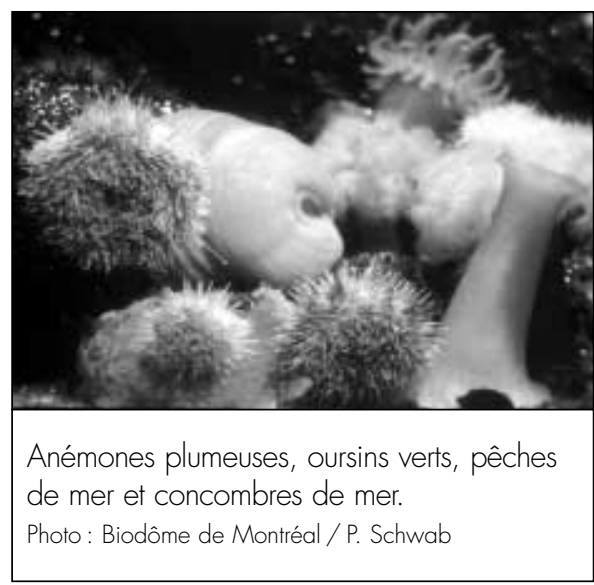

particulier sur des réseaux de solidarités parentale, culturelle et relationnelle fortes, de type «clanique », - a maxima -, ou « clientéliste », - a minima -, notamment).

$\mathrm{Si}$, en effet, seule une partie du groupe considéré bénéficie de la mise en œuvre et des retombées économiques du produit et de l'activité en cause, cela aura pour conséquence d'engendrer à son profit une « rente de situation», au détriment des autres groupes ou membres de la communauté. Il en résulte des situations inégalitaires et donc des tensions, des rivalités, voire des potentialités de conflits, sources d'autant de facteurs de déstructuration de la communauté, de destruction des solidarités originaires et de rupture de la cohésion sociale. Les bénéfices pour la communauté risquent donc d'être à terme surtout négatifs, au-delà des seules retombées économiques immédiates et apparentes.

Cela suppose alors que les populations réceptives soient, à la fois singulièrement et globalement, appelées à réoccuper une place centrale essentielle dans le fait touristique, dans l'offre de produits, dans l'organisation et la maîtrise des politiques, dans la réponse à la demande, afin qu'elles deviennent des acteurs et des décideurs à part entière d'une activité qu'elles n'auront plus à subir passivement.

Il leur appartiendra au contraire, en synergie avec les différents partenaires publics et privés, de la déterminer, de l'orienter et de la contrôler, en redéfinissant le tissu relationnel qu'elle postule et en contribuant à proposer des produits adaptés, à partir d'un processus conjoint de sensibilisation, de mobilisation et de responsabilisation.

\section{L'illusion du développement univoque}

Il faut se garder de considérer que le tourisme, en général, et l'écotourisme, en particulier, constitueraient la seule alternative crédible au financement du développement économique dans les DOM-TOM, face au déclin des productions agricoles, au faible potentiel industriel et à l'exploitation encore embryonnaire des gisements de services. Les courbes de convergence du développement du tourisme et de la productivité économique tendent en effet à s'aplatir assez rapidement, au bout de quelques années, illustrant par-là les limites de la capacité du tourisme à dynamiser la croissance au-delà d'un seul effet temporaire d'accélération, pendant une période initiale ${ }^{24}$.

Le danger est en effet de voir les initiatives et les efforts, et donc les investissements, être détournés de ce fait, par un effet d'écran, d'autres secteurs d'activités, également porteurs de potentialités de développement durable, en raison d'une attente excessive, sinon quasi-exclusive, à l'endroit du seul secteur touristique.

Plus généralement, l'idée d'enracinement (roots...), inhérente à une vision du développement nécessairement ancrée dans le vécu local, et dûment mise au service de celui-ci, renvoie à une dimension patrimoniale foncière indissociable de l'histoire douloureuse et chaotique de la plupart des peuples qui habitent aujourd'hui les DOM, dans la Caraïbe en particulier. Au carrefour de l'histoire et de l'anthropologie, on y verra un paramètre essentiel de la problématique écotouristique et de sa maîtrise par les populations et les communautés résidentes, à travers l'analyse des représentations et des pratiques qui président aujourd'hui à l'occupation et à la gestion des espaces.

\section{La propension à l'« a-culturation »}

Face à un tourisme destructeur du fait de l'importation sans nuance de comportement et de valeurs exogènes, support d'une démarche économiquement mercantile et socialement déstructurant, les populations locales tendent à perdre la maîtrise de leurs espaces et de leurs modes de vie, et donc de leurs valeurs identitaires, qu'il leur appartient de se réapproprier.

L'écotourisme, dans cette acception très originale et paradoxale, procéderait alors d'un processus d'accueil, de rencontre, de découverte et de partage mutuel, plus positivement «acculturant», dans sa dimension syncrétique et intégrante, qu' «a-culturant », en termes de destruction des valeurs et des modes de vie locaux.

Il n'apparaît pas plus utopique de rechercher une indispensable cohérence, en termes tout autant culturels que juridiques, entre identité, territoire (terroir?) et patrimoine environnemental. On peut mettre en évidence la considération que les représentations et les pratiques liées à la perception des paysages domestiqués ou naturels participent de l'identité locale, l'espace personnel et habité étant de ce point de vue largement approprié, géré, maîtrisé et symbolisé.

La référence au sol et l'appropriation de l'espace, comme la transmission corrélative du patrimoine, procèdent fondamentalement en ce sens d'une aspiration identitaire forte qui renvoie à la quête des origines.

\section{Les ambiguïtés de la réappropriation identitaire}

La référence identitaire, en termes de « reconstruction » et de « réappropriation », appelle, si l'on entend nuancer et affiner le propos, quelques observations complémentaires qui exigeraient d'être par ailleurs développées et approfondies. On se limitera pour cette raison aux remarques ci-après, qui ne prétendent proposer que quelques pistes incontournables de réflexion ${ }^{25}$.

\section{Identité et authenticité}

i) Parmi les offres d'écotourisme figure au premier plan toute la gamme des spectacles et des manifestations, qui puisent leurs racines dans les traditions et les cultures locales. Ils constituent des produits d'appel importants et des composantes attractives de l'offre touristique. Dans les destinations exotiques, ils font souvent l'objet d'une inévitable dénaturation et ce, dans presque tous les cas de figure, quelles que soient les précautions que l'on entende prendre ou la vigilance dont on entende faire preuve à cet égard. Il en va ainsi, de manière manifeste, lorsqu'il s'agit de produits conçus et mis en place pour les touristes, eu égard à leurs attentes et à leurs comportements supposés ${ }^{26}$. Leur défaut d'authenticité leur confère de ce fait une valeur quasi nulle, en termes de réappro- 
priation comme de partage culturels, pour le touriste aussi bien, a fortiori, que pour le détenteur originaire de la culture ainsi artificiellement proposée et exposée.

C'est également le cas, dans une moindre mesure, de manifestations traditionnelles réellement authentiques, mais qui, à partir du moment où elles se déroulent avec (i.e. en présence) des touristes et leur sont ouvertes, seraient-ils peu nombreux, ne peuvent plus être perçues ni vécues de la même façon par les populations autochtones. L'effet de dénaturation, bien que plus réduit et latent, n'en est pas moins présent, du fait à la fois de la rupture implicite, mais inévitable, de la cohésion culturelle et psychologique du groupe et de la finalité inévitablement pervertie de la démarche.

ii) Il convient également de savoir si la demande, en matière d'écotourisme, provient bien des communautés et des populations locales ou si elle ne leur est pas au contraire plus ou moins artificiellement suggérée, sinon même imposée, et qu'elle est dûment encadrée à cet effet. La situation n'est en effet pas la même selon que la démarche écotouristique a une origine exogène, construite de l'extérieur, quitte à être réappropriée par le groupe s'il l'estime pertinente et profitable, a fortiori si elle s'avère réellement bénéfique, ou qu'elle procède de la perception de besoins comme de la formulation conséquente de demandes spécifiques par le groupe lui-même et à sa seule initiative, de manière endogène, à partir de sa culture et de ses valeurs identifiées à travers ses propres référents ${ }^{27}$.

\section{Identité et extranéité}

i) Quand on évoque la « réappropriation » de valeurs identitaires, il est en outre nécessaire de nuancer le propos, car il s'agit souvent, en réalité, d'appropriation plus que de réappropriation. Il y a bien en effet réappropriation lorsqu'il s'agit d'un patrimoine originaire et propre du groupe ou de la communauté, à l'endroit d'une partie de son héritage historique (cimetières d'esclaves, par exemple, gisements archéologiques ou traditions magiques).
À l'inverse, lorsqu'il s'agit d'intégrer au patrimoine local, et d'en revendiquer la dimension culturelle, des vestiges architecturaux ou monumentaux issus d'une activité économique ou militaire qui était celle du colonisateur (ce qui représente souvent la grande majorité, sinon la quasi-totalitéé du patrimoine immobilier et bâti : moulins de broyage de canne, « habitations » des anciens domaines fonciers, églises importées avec le culte du colonisateur, forts et bastides, etc.), il ne saurait s'agir que d'appropriation (initiale) par une communauté différente de celle à laquelle est due la constitution du patrimoine en cause.

ii) Souvent, également, la notion de terroir et de patrimoine endogène reste limitée à ce qui relève du domaine de la propriété et des relations privées, voire traditionnelles. «Ce qui est à l'État » (Biti a léta, cé ta vou ? : ce qui appartient à l'État, est-ce que c'est à vous ?) (Rauzduel, à paraître), dans les $\mathrm{DOM}^{28}$ notamment, n'est ni perçu ni en conséquence revendiqué comme tel. Cela suffit paradoxalement à justifier des comportements de désintérêt et de désinvolture, voire d'ignorance ou de rejet, à l'opposé de tout souci de préservation, de conservation, a fortiori de réappropriation, s'agissant de biens (corporels et incorporels) relevant de la chose publique qui demeurent, par l'effet d'une telle perception «négatrice», extérieurs au noyau culturel et patrimonial.

iii) Il faut, enfin, demeurer réservé et prudent envers les parfois prétendues traditions culturelles, sur lesquelles asseoir un projet écotouristique. Elles ne trouvent en effet souvent leur origine que dans une récupération, voire une reconstruction par une frange bourgeoise des sociétés, apte à accéder à une culture et à une démarche intellectuelle qui sont celles d'une certaine élite. Procédant de leur seule démarche volontariste (serait-elle implicite dans la durée, au terme d'un processus diachronique peu aisé à appréhender), faute de laquelle elles n'auraient pas été ainsi (implicitement, mais sans doute insidieusement) révélées ou mises à jour, elles restent par-là, une fois encore, extérieures aux masses populaires, que l'on entend alors persuader qu'il s'agit de essentielles de leur patrimoine...
Identité et durabilité

i) Est-il donc encore possible, dans le contexte des DOM-TOM, de la Réunion à la Guadeloupe, de Mayotte à la Martinique, du Pacifique à la Guyane, de prétendre réconcilier tourisme et environnement, tourisme et culture, tourisme et hospitalité (au sens étymologique fort) ? Activités touristiques et exigences environnementales seraient-elles par essence incompatibles, et donc irréductibles, au point de vouer au conflit perpétuel et à la rupture dans l'autodestruction le « couple d'enfer» (selon le mot d'un journaliste) constitué par le tourisme et l'environnement ? Serait-ce alors « un rêve que d'envisager un mode de tourisme respectueux de l'espace »?

Selon un observateur averti, «la reconnaissance du tourisme durable suppose conjointement une internationalisation des besoins et des ressources et une intégration généralisée et systématisée de l'environnement dans l'ensemble des politiques et des économies » (Blouin, 2000b : 42), sauf, pour celui-ci, qu'il faut éviter de confondre tourisme durable et écotourisme et de purement et simplement, voire abusivement, l'y assimiler.

L'objectif réside, par l'intégration élargie de l'environnement au tourisme, dans l'élaboration et la gestion d'un développement touristique dûment encadré et maîtrisé qui devrait, à cet effet, appréhender le tourisme dans sa globalité, sur les plans local, national et international, par une approche qui intègre la protection de l'environnement et les droits de l'Homme à un environnement sain et durablement protégé.

ii) On peut en ce sens, au terme de ce propos, risquer une nouvelle définition de l'écotourisme, davantage conforme et appropriée à la mise en valeur des ressources patrimoniales et environnementales des DOM-TOM. On y verra ainsi, au-delà peut-être du seul tourisme écologiquement responsable ou solidaire que retiennent pour leur part certains (de notre point de vue un peu trop réducteurs, bien que non dépourvus d'arguments) ou, dans une perspective sensiblement différente, du tourisme finaliste et différencié, à objectifs culturels et altruistes, auquel d'autres peuvent se rallier, un tourisme de «terroir», terroir à la fois culturellement partagé, réapproprié et collectivement protégé et valorisé. 
L'écotourisme serait ainsi une composante originale, parfois déterminante, d'un tourisme durable garant d'un développement viable. Il peut alors constituer, dans le contexte particulier de certaines des régions ultra-périphériques françaises et, eu égard à leur histoire comme à leur avenir, à la fois le lieu, l'occasion et l'opportunité d'une nécessaire « reconstruction » identitaire, à partir d'une réappropriation patrimoniale et, partant, d'une valorisation socioculturelle des terroirs et des ressources de biodiversité.

\section{Conclusion}

Ce constat, comme première approche d'une réflexion à développer et à approfondir, n'a d'autre prétention que de verser une pièce supplémentaire à un débat complexe, qui est celui de la recherche de réponses appropriées et efficientes aux handicaps, aux faiblesses et aux insuffisances du tourisme traditionnel dans certains DOM, antillais en particulier.

Ceux-ci s'expriment respectivement, et de manière cumulée, si l'on se réfère à l'exemple actuel de la Guadeloupe, sur plusieurs plans ; c'est le cas à la fois des plans structurel et infrastructurel (insuffisance de l'offre de transport aérien au regard de la capacité hôtelière et non-renouvellement et inadaptation du parc hôtelier), socioculturel (rejet latent d'une occupation exogène des espaces et croissance de l'insécurité), politico-économique (surcoûts de la main-d'œuvre et grèves récurrentes) et médiatico-commercial (inadaptation de l'offre et insuffisance de sa diversification et de sa médiatisation).

La question se pose, en fin de compte, de savoir si, et dans quelle mesure, le défi de l'écotourisme pouvait et serait en situation de répondre en partie à certains des handicaps et des faiblesses du tourisme traditionnel de masse, dans les destination de soleil des DOM-TOM. Il est certes conduit, a priori, à se heurter aux mêmes obstacles, dans leur composantes endémiques. Mais, bien qu'il ne constitue (et n'a par essence vocation qu'à constituer) qu'une niche relativement marginale, bien qu'en développement significatif, de l'offre de tourisme, il n'en est pas moins apte à réduire, sinon à contourner, certains d'entre eux, en proposant une offre alternative crédible bien que limitée.
Il conviendrait alors, pour les DOM-TOM, si cela est avéré, et en partant d'un état des lieux approprié, d'un bilan objectif des expériences conduites et d'une évaluation exhaustive et réaliste des potentialités et des conditionnalités, d'en cibler l'objet et d'en finaliser le contenu, nécessairement spécifiques pour chacun d'entre eux (en raison de la diversité de leurs potentiels, mais également dans le but d'atténuer les handicaps d'une concurrence inopportune ou inégale), afin d'en valoriser l'exploitation au service d'une développement local viable.

Il n'est pas exclu, au demeurant, si l'on entend in fine élargir le champ de l'analyse, que la signification et la portée de ces propos puissent être validées à l'endroit d'autres régions du monde où, au-delà de l'écart et des spécificités constatés dans les données de l'environnement politique, économique, social et culturel, les sociétés et les communautés présentent une sensibilité sinon une vulnérabilité comparables vis-à-vis de l'expansion du phénomène et de la pression touristiques.

La crédibilité et l'effectivité du paradigme d'écotourisme, pour autant qu'il y soit vérifié et dans la mesure de ceux de ses paramètres pour lesquels il le serait, pourrait alors utilement contribuer à déterminer et à élaborer des offres différentes de tourisme. Celles-ci pourraient ainsi s'avérer davantage conformes aux attentes des États en cause, en termes de développement touristique durable favorable à la préservation et, peut-on l'espérer, à l'optimisation pérenne des valeurs inhérentes à l' «être» des sociétés en devenir.

Jean-Marie Breton est professeur à l'Université des Antilles et de la Guyane (UFR des Sciences Juridiques et Économiques de la Guadeloupe), directeur du CREJETA (Centre de Recherches et d'Études Juridiques sur l'Environnement, le Tourisme et l'Aménagement).

\section{Notes}

1 Départements et Territoires d'outre-mer de la Caraïbe, de l'Océan Indien et du Pacifique.

2 Voir, entre autres, Breton (2000 : 13-24).

3 Notion qui ne se confond pas avec la notion de tourisme durable, même si elle la recoupe largement, telle qu'elle a été notamment précisée par l'Organisation mondiale du tourisme (OMT), à travers celle de développement touristique durable.

4 Voir Portfolio of Statements \& Presentations (CD-ROM / OMT, 2002 ; documents également accessibles sur les sites suivants : [www.uneptie.org/tourism] et [www.world-tourism.org].

5 Voir les remarques nuancées formulées en ce sens par M. Couture et rapportées plus loin.

6 On se réfèrera, avec le plus grand intérêt, au Dossier «Écotourisme » qui figure dans une récente livraison de cette Revue (Téoros, vol. 21, $\mathrm{n}^{\circ} 3$, automne 2002), en particulier aux réflexions et aux analyses présentées par Maurice Couture (2002a: 3-4; et 2002b : 5-13). Selon cet auteur, l'écotourisme prendrait diverses formes, étant à la fois, ou alternativement, un produit ou une expérience touristique, un segment ou une niche de marché, une forme de tourisme ou de voyage, un secteur ou un sous-secteur du tourisme, une approche de développement et de gestion, un laboratoire de tourisme durable en milieu naturel (2002a: 3). En tout état de cause, l'écotourisme, en ce qu'il se distingue d'autres formes de tourisme «alternatif», "est clairement une composante du tourisme de nature, peut-être jumelé dans le cadre d'un séjour à d'autres formes compatibles de tourisme, et constitue une manifestation évidente de 'tourisme durable' qui concerne toutes les formes de tourisme, qu'il s'agisse de tourisme urbain, rural ou de nature» (2002b : 7).

7 «Modèle théorique de pensée qui oriente la recherche et la réflexion scientifiques » (Petit Larousse, 2000).

8 Christiane Gagnon (1999) ne se réfère pas systématiquement, ni explicitement, au concept d'écotourisme, même si celui-ci demeure largement sous-jacent aux présupposés et aux orientations de sa réflexion. C'est plutôt à propos de la clarification des enjeux scientifiques du « développement durable et viable» (que nous abordons plus loin) que cette auteure évoque expressément un "paradigme novateur », l'écotourisme n'en apparaissant alors que comme un avatar opérationnel, parmi d'autres.

9 Nous estimons, pour notre part, que l'écotourisme, faute de «définition» possible ou crédible, repose à tout le moins sur trois paramètres fondamentaux et déterminants, procédant des concepts de nature, de responsabilité et de durabilité. Ceux-ci se déclineraient alors respectivement en termes de gestion environnementale et patrimoniale, de valorisation culturelle et identitaire partagée et de développement local participatif et intégré. 
10 Lequin (2001: 4); celui-ci précise plus loin, en ce sens, "Nous concevons une perspective d'analyse de l'écotourisme comme un système d'action et de décision où les protecteurs de la ressource, les promoteurs touristiques ainsi que les communautés locales seront considérés comme des acteurs à part entière du processus de développement et de mise en valeur d'un site écotouristique à caractère unique» (2001 : 11).

11 Voir infra le débat sur le pluralisme conceptuel de la référence à l'écotourisme; voir également, M. Lequin, 2001 : 14-18.

12 Cf. infra. Marie Lequin nuance d'ailleurs elle-même quelque peu son propos, lorsqu'elle relativise la valeur même du concept, en reconnaissant que c' " est un concept ambigu qui, selon le point de vue adopté, se définit comme une activité, une philosophie ou une stratégie de développement» (2001: 13).

13 Tout cela est excellemment développé dans l'ouvrage précité de Lequin, (2001 : 27-40).

14 Entre autres multiples définitions, voir celles mentionnés par S. Blangy (1993) et F. Bouin (2000). Ibid., dans NATUROPA, Conseil de l'Europe, 1997.

15 Le débat est d'ailleurs, de manière significative, de plus en plus fréquent dans la Caraïbe (voir à ce sujet: Carnegie et Anderson, 1998 ; Breton, 1999).

$16 \mathrm{~S}$. Blangy et F. Kouchner parlent, à juste titre, à propos du terme d'écotourisme, d'un «glissement sémantique qui souligne la dimension résolument sociale, voire politique, de la plupart des initiatives» (2002: 23).

17 Pour M. Lequin, «sur un plan [...] individuel, l'écotourisme peut être analysé comme la recherche d'une expérience d'interaction affective et consciente avec un certaine forme ou activité de nature », permettant de I'analyser "dans une perspective phénoménologique» (dans L'écotourisme. Expérience d'une interaction nature-culture, ibid., p. 42).

18 Entre autres, les diverses études consacrées au tourisme, à l'environnement et à l'écotourisme, dans «Tourisme et environnement - Du tourisme de nature à l'écotourisme», Les Cahiers d'Espaces, février 1993 (en particulier, S. Blangy, «L'écotourisme dans le monde», p. 223).

19 Englobant, dans une acception extensive, non seulement les ressources naturelles biotiques et abiotiques, mais aussi le cadre et la qualité de vie, les traditions et les valeurs culturelles, le patrimoine historique, architectural et archéologique, etc. ( $c f$. infra).

20 Voir le numéro de Téoros consacré aux «Patrimoines du $\mathrm{XX}^{\mathrm{e}}$ siècle», 2002, vol. 21, n 2.
21 On peut craindre, comme il a été alors aisé de le constater, que la logique étroitement «économiciste » ait implicitement pris le pas - ou à tout le moins y ait occupé une place prédominante - sur les autres considérations et paramètres lors du Sommet mondial de Québec, dès lors que les opérateurs privés de tourisme y ont été très présents et actifs, ont largement participé aux divers travaux et débats et ont entendu que leur logique soit dûment prise en compte dans les constats et les recommandations intégrés dans la déclaration finale... (sur ce texte et le contexte dans lequel il a été élaboré, voir Lemaistre, 2002 : 14-19).

22 Opinion que partage manifestement $\mathrm{M}$. Couture quand il souligne que «l'utilisation du terme écotourisme fait souvent office de fourre-tout» $(2002 \mathrm{~b}: 10)$.

23 Notre analyse rejoint sur ce point, serait-ce par une autre approche, celle de M. Lequin pour qui «l'écotourisme s'inscrit inexorablement dans une relation particulière entre nature et culture au sein d'une société, soit un niveau qui met en évidence une dimension anthropologique», de telle sorte que « si l'écotourisme est culturellement déterminé, il en serait de même, à l'évidence, en ce qui regarde les produits et les retombées de l'écotourisme qui doivent être développés en congruence avec les cultures et qui ne sont pas a priori de caractère universel» (2001:42).

24 B. Marques (à paraître) conclut que «le tourisme serait durable [sur le plan économique] dans la mesure où il contribuerait au démarrage économique d'un pays ».

25 Pour des développements approfondis sur cette question particulièrement complexe, dans sa dimension culturelle, notamment, et dans l'espace caribéen «français » en particulier, voir Reno (1995) et Constant (2000).

26 Voir Téoros, dossier «Comportements touristiques », 2001, vol. 20, n 3 et, notamment, la présentation de J. Stafford en page 4.

27 Dans l'esprit des deux précédentes remarques, on attirera parallèlement l'attention sur le phénomène de «doudouïsme» (le doudou est la personne aimée ; par extension le nom donné au touriste interpellé et choyé par les mamas commerçantes des marchés), bien connu dans les Antilles françaises (à Marie-Galante, par exemple), dont peut également pâtir l'écotourisme. Il consiste, pour les concepteurs et les porteurs locaux de produits artisanaux et culturels, à proposer des produits et des activités fondés sur des images artificiellement « reconstruites », à partir de l'identification a priori de schémas et de comportements estimés aptes à répondre à l'attente supposée des touristes, sur laquelle, pour des raisons d'efficacité et de rentabilité, il paraît pertinent de s'aligner.

28 Expression relevée en Guadeloupe notamment.

\section{Bibliographie}

Blangy, C. (1993), «L'écotourisme dans le monde », Les Cahiers d'Espaces, février, p. 223.

Blangy, S., G. Dubois, et F. Kouchner (2002), «Écotourisme. Expériences françaises », Cahiers de l'AFIT, Paris.

Blangy, S., et F. Kouchner (2002), «Produits, destinations, particularités et enjeux de l'écotourisme en Europe », Téoros, vol. 21, n 3, p. 23.

Blouin, F. (2000a), «L'écotourisme, au carrefour du tourisme et de l'environnement», dans L'écotourisme: un nouveau défi pour la Caraïbe?, Paris, Karthala, Colloque «Iles et pays d'outre mer », vol. 1, p 25-32.

Bouin, F. (2000b), Tourisme et droit de l'environnement, thèse, Limoges, janvier, document polycopié, p. 412.

Breton, J.M. (2000), «L'écotourisme: une problématique conflictuelle pour des politiques innovantes », dans L'écotourisme: un nouveau défi pour la Caraïbe?, Paris, Karthala, Colloque «Îles et pays d'outre mer», vol. 1, p. 13-24.

Breton, J.M. (1999), «Qu'est-ce que l'écotourisme? », dans L'écotourisme, une chance pour le tourisme des DOM, Troisièmes Assises nationale des OT et SI des DOM, Cayenne, mai.

Carnegie, A.R., et W.C. Anderson (1998), The Legal Framework of Biodiversity: A West Indian Perspective, Caribbean Workshop on Marine Biodiversity, Montego Bay, Jamaica, octobre 1998, document polycopié, 13 p.

Couture, Maurice (2002a), «L'écotourisme en 2002 et après...», Téoros, vol.21, n³, automne, p. 3-4.

Couture, Maurice (2002b), «L'écotourisme, un concept en constante évolution », Téoros, vol.21, $\mathrm{n}^{\circ} 3$, automne, p. 5-13.

Constant, F. (2000), Le Multiculturalisme, Flammarion, Paris.

Gagnon, Christiane (1999), Tourisme viable et parcs nationaux: Quel avenir pour les collectivités locales?, Chicoutimi, UQAC.

Kurt, K. (1993), «Le concept d'écotourisme», Les Cahiers d'Espaces, février, p. 214.

Lequin, Marie (2001) Écotourisme et gouvernance participative, PUQ, Montréal.

Lemaistre, Ph. (2002), «Année internationale de l'écotourisme. Un premier bilan », Téoros, vol. 21, n 3, p. 14-19.

Marques, B. (à paraître), Tourisme durable et croissance: L'expérience des pays de la Caraïbe sur les vingt dernières années, Actes du Colloque «Tourisme et développement durable», Fort-de-France, Martinique, septembre 2003.

Py, P. (2000), «Du tourisme écologique à l'écotourisme», Tourisme et Droit, ${ }^{\circ} 18$, mai, p. 41.

Rauzduel, R. (à paraître), «De la difficulté d'être participant écologique sur les aires touristiques en Guadeloupe. Les Chutes du Carbet», dans J.M. Breton (dir.), Tourisme, environnement et aires protégées, Karthala, Paris.

Reno, F. (1995) (dir.), «Identité et politique. De la Caraïbe et de l'Europe multiculturelles », Economica, Paris. 